\title{
Use of Organic Precursors and Graphenes in the Controlled Synthesis of Carbon-Containing Nanomaterials for Energy Storage and Conversion
}

\begin{tabular}{|r|l|}
\hline Journal: & Accounts of Chemical Research \\
\hline Manuscript ID: & ar-2012-001475.R2 \\
\hline Manuscript Type: & Article \\
\hline Date Submitted by the Author: & 03-Oct-2012 \\
\hline Complete List of Authors: & $\begin{array}{l}\text { Yang, Shubin; Max Planck Institute for Polymer Research, Max-Planck- } \\
\text { Institut fur Polymerforschung Mainz, Germany, } \\
\text { Bachman, Robert; The University of the South, Department of Chemistry } \\
\text { Feng, Xinliang; Max-Planck Institute for Polymer Research, Synthetic } \\
\text { Chemistry } \\
\text { Mullen, Klaus; Max-Planck-Institute for Polymer Research, }\end{array}$ \\
\hline
\end{tabular}




\title{
Use of Organic Precursors and Graphenes in the Controlled Synthesis of Carbon-Containing Nanomaterials for Energy Storage and Conversion
}

\author{
Shubin Yang ${ }^{+}$, Robert E. Bachman ${ }^{+}$Xinliang Feng ${ }^{+\#, *}$, Klaus Müllen $^{+, *}$ \\ ${ }^{+}$Max Planck Institute for Polymer Research, Ackermannweg 10, D-55128 Mainz, Germany \\ Department of Chemistry, The University of the South, 735 University Avenue, 37383 , \\ Sewanee, TN, USA \\ \# School of Chemistry and Chemical Engineering, Shanghai Jiao Tong University, 200240, \\ Shanghai, P. R. China
}

KEYWORDS: Graphene, nanomaterials, supercapacitor, lithium ion batteries, fuel cells

\section{CONSPECTUS}

The development of high-performance electrochemical energy storage and conversion devices, including supercapacitors, lithium-ion batteries and fuel cells, is an important step on the road to alternative energy technologies. Carbon-containing nanomaterials (CCNMs), defined here as pure carbon materials and carbon/metal (oxide, hydroxide) hybrids with structural features on the nanometer scale, show potential application in such devices. Because of their pronounced 
electrochemical activity, high chemical/thermal stability and low cost, researchers are interested in CCNMs to serve as electrodes in energy-related devices.

Various all-carbon materials are candidates for electrochemical energy storage and conversion devices. Furthermore, carbon-based hybrid materials, which consist of a carbon component with metal oxide or metal hydroxide-based nanostructures, offer the opportunity to combine the attractive properties of these two components and tune the behavior of the resulting materials. As such, the design and synthesis of CCNMs provide an attractive route for the construction of high-performance electrode materials. Studies in these areas have revealed that both the composition and the fabrication protocol employed in preparing CCNMs influence the morphology and microstructure of the resulting material and its electrochemical performance. Consequently, researchers have developed several synthesis strategies, including hard-templated, soft-templated, and template-free synthesis of CCNMs.

In this Account, we focus on recent advances in the controlled synthesis of such CCNMs and the potential of the resulting materials for energy storage or conversion applications. The Account is divided into four major categories based on the carbon precursor employed in the synthesis: low molecular weight organic or organometallic molecules, hyperbranched or cross-linked polymers consisting of aromatic subunits, self-assembling discotic molecules, and graphenes. In each case, we highlight representative examples of CCNMs with both new nanostructures and electrochemical performance suitable for energy storage or conversion applications. In addition, this Account provides an overall perspective on the current state of efforts aimed at the controlled synthesis of CCNMs and identifies some of the remaining challenges. 


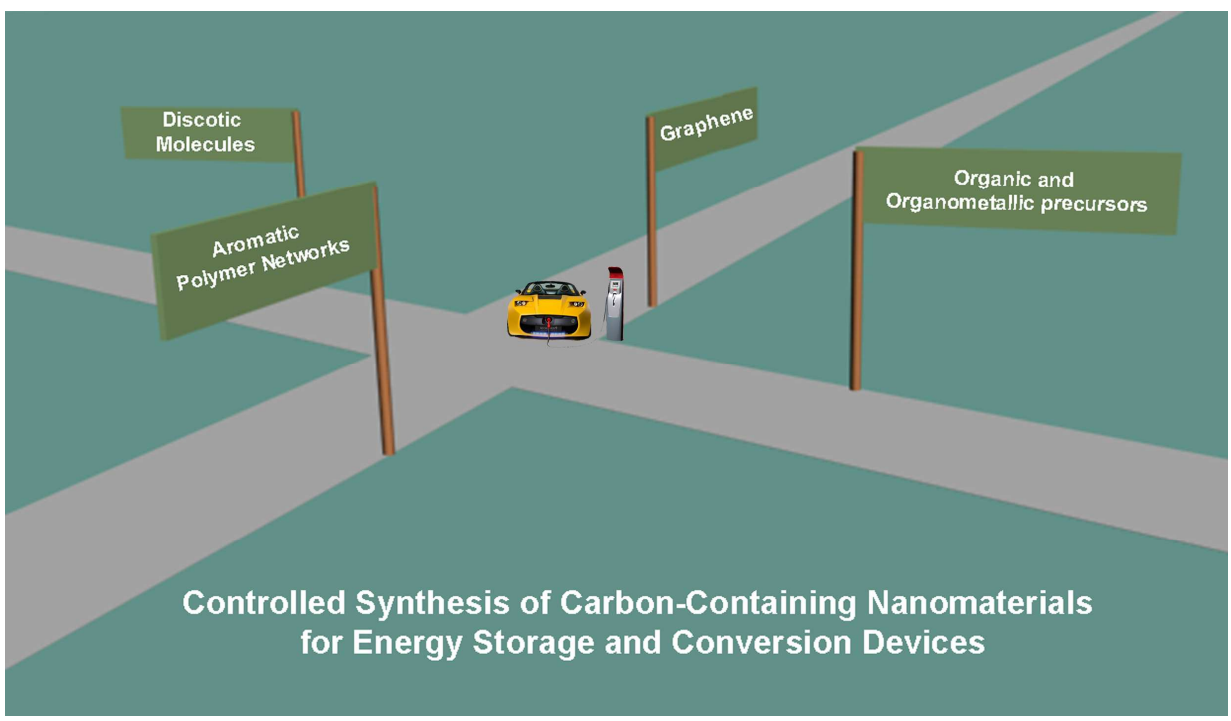

TOC

\section{Introduction}

Fossil fuel depletion and global climate change present society with environmental and energy sustainability challenges. ${ }^{1,2}$ In response to these challenges there has been a strong push toward the adoption of electric vehicles (EVs) and hybrid EVs (HEVs) for transportation. However, a shift to such vehicles will require the development of more efficient portable energy storage and conversion devices. ${ }^{1-4}$ Due to their energy and power densities, rechargeable lithium-ion batteries, supercapacitors/ultracapacitors and fuel cells are promising candidates to meet this need. ${ }^{2}$ Since the performance of these devices depends intimately on the nature of the electrode materials, the development of new electrochemically active materials with high activities and robust structures has become an area of considerable research interest. ${ }^{5,6}$

Pure carbon materials and carbon/metal (oxide, hydroxide) hybrids with nanometer scale structures, collectively referred to in this Account as carbon-containing nanomaterials (CCNMs), have become attractive as electrodes in energy-related devices because of their pronounced electrochemical activity, good electrical conductivity, high chemical/thermal stability and low 
cost. $^{7-9}$ Given the relationship between structural features and bulk properties in these materials, it is worth clearly defining key structural terms at the outset. Specifically, the carbon component in CCNMs can be categorized as either crystalline or amorphous based on the degree of long-range structural order present. In the context of the electrode materials considered here, amorphous carbon may be defined as consisting of local graphitic domains dispersed in a carbon matrix with ill-defined long-range order.

Various all-carbon materials, with graphitic or amorphous structures, have been suggested as candidates for electrochemical energy storage and conversion devices. ${ }^{7-10}$ Furthermore, carbon-based hybrid materials, which combine a carbon component with metal (oxide or hydroxide)-based nanostructures, ${ }^{11,12}$ offer the opportunity to combine the attractive properties of the two components and thereby tune the behavior of the resulting materials for enhanced device performance. As such, CCNMs seem to provide an attractive route for the construction of high-performance electrode materials. ${ }^{7,13-15}$ Studies in these areas have revealed that not only the composition but also the fabrication protocol employed in preparing CCNMs exerts a large influence on the resulting electrochemical performance owing to its impact of the morphology, microstructure and composition of the resulting material. ${ }^{16}$ Consequently, several strategies such as hard-templated ${ }^{17}$, soft-templated ${ }^{13}$ and template-free synthesis ${ }^{15}$ of CCNMs have been developed. In this Account, we summarize recent achievements, primarily focusing on our contributions, toward the controlled synthesis of CCNMs for energy storage and conversion. This Account is divided into four major sections guided by the type of precursor employed in the preparation of the CCNMs: low molecular weight organic and organometallic precursors, ${ }^{18,19}$ 
hyperbranched or cross-linked polymers with built-in aromatic subunits (defined as aromatic polymer networks here), ${ }^{15,20}$ discotic molecules capable of pre-organizations in the solid or liquid-crystalline state, ${ }^{7,21}$ and graphene sheets with varying degrees of oxidation. ${ }^{12,13,22}$

\section{Low molecular weight organic and organometallic precursors}

(a)

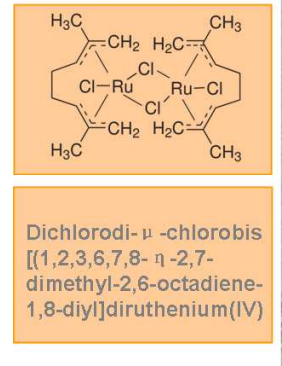

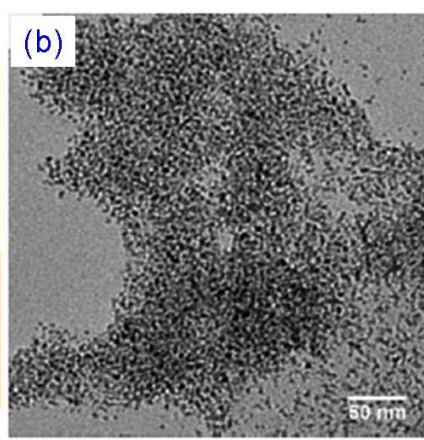

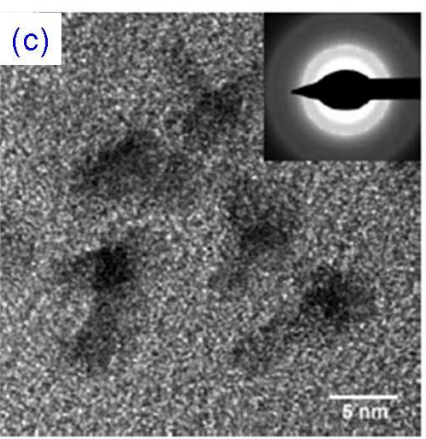

FIGURE 1. (a) Chemical structure of the Ru-containing precursor. (b) TEM and (c) HRTEM images of the carbon-Ru composite, with a selected area electron diffraction pattern shown in the insert. $^{23}$

Low molecular weight precursor molecules can be converted into CCNMs via pyrolysis, with or without the use of an additional structured template. While the chemical nature of the precursor employed in the pyrolysis process plays a dominant role in determining the composition, structure and function of the final CCNM, additional factors, such as the presence or absence of a template and the processing conditions, also play a role.

In 2007, we reported the pyrolysis of dichlorodi- $\mu$-chlorobis $[(1,2,3,6,7,8$ -

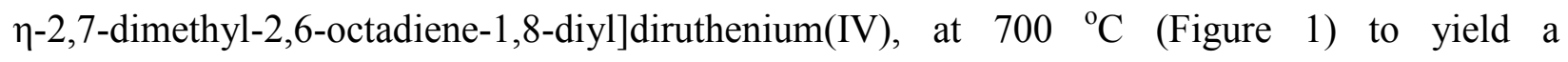
carbon- $\mathrm{Ru}$ composite. This CCNM was composed of crystalline Ru nanoparticles (3-7nm in diameter) homogeneously dispersed in a mesoporous amorphous carbon matrix. ${ }^{23}$ The $\mathrm{Ru}$ 
nanoparticles could subsequently be converted into $\mathrm{RuO}_{2}$ via electro-oxidization in a sulfuric acid solution. The resulting carbon- $\mathrm{RuO}_{2}$ composite functioned as supercapacitor, with a capacitance of $132 \mathrm{~F} \mathrm{~g}^{-1}$ in $0.5 \mathrm{M} \mathrm{H}_{2} \mathrm{SO}_{4}$ electrolyte. ${ }^{23}$ This value is comparable to that reported for carbon- $\mathrm{RuO}_{2}$ composites prepared via a more complex multistep colloid-based synthesis. ${ }^{24}$

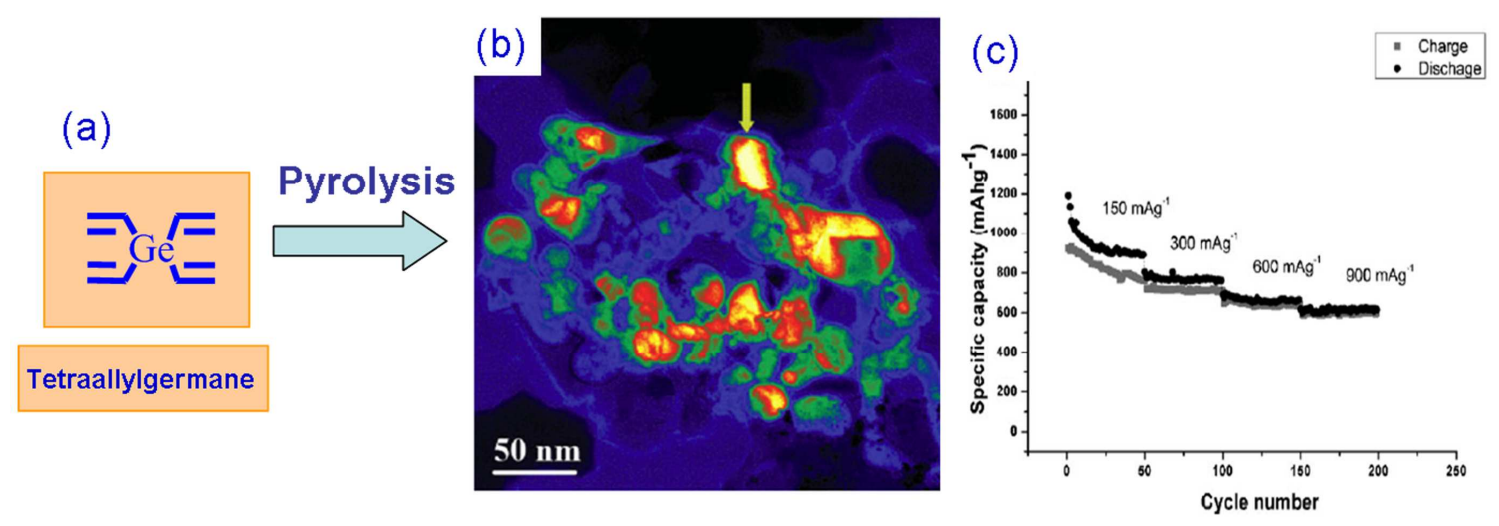

FIGURE 2. (a) Chemical structure of the tetraallylgermane precursor. (b) Elemental analysis of carbon-Ge composite. (c) Cycle and rate performances of carbon-Ge composite. ${ }^{18}$

Similarly, pyrolysis of other organometallic precursors, such as allyltriphenyltin ${ }^{19}$, tetraallylgermane $^{18}$ or coordination complexes, such as cobalt acetylacetonate ${ }^{25}$, also yield CCNMs (Figure 2). For example, pyrolysis tetraallylgermane generated a CCNM with 50 to 70 nm Ge nanoparticles homogeneously dispersed in an amorphous carbon matrix. ${ }^{18}$ The resulting carbon-Ge composite exhibited a reversible and stable charge capacity of approximately $900 \mathrm{~mA}$ $\mathrm{h} \mathrm{g}^{-1}$ at a current density of $150 \mathrm{~mA} \mathrm{~g}^{-1}$. Furthermore, a capacity of $613 \mathrm{mAh} \mathrm{g}^{-1}$ was achieved at the higher current density of $900 \mathrm{~mA} \mathrm{~g}^{-1}$ (Figure 2c), which is much higher that of electrodes contain only Ge nanoparticles $\left(\sim 200 \mathrm{mAh} \mathrm{g}^{-1}\right) .{ }^{18}$ The excellent electrochemical performance of this carbon-Ge composite can be attributed to several structural features. The small size of the Ge 
nanoparticles reduces the lithium diffusion length during the cycling process to a few nanometers. Additionally, the carbon matrix maintains a good electrical conductivity in the electrodes while providing mechanical support and thereby preventing disintegration and/or aggregation of the metal particles.

Despite the successful synthesis of various CCNMs via the template-free pyrolysis of organometallic and coordination complex precursors, control of the bulk morphology as well as the meso- and nano-scale structure of the CCNM products remains limited. ${ }^{18,23}$ In an attempt to overcome these limitations, the use of inorganic templates during pyrolysis has been developed in recent years. For instance, pyrolysis of butylcapped silicon in a SBA-15 silica template at $900^{\circ} \mathrm{C}$ generated mesoporous $\mathrm{Si} @ \mathrm{C}$, consisting of $\mathrm{Si} @ \mathrm{C}$ nanowires $(6.5 \mathrm{~nm})$ and ordered mesopores $(2.3 \mathrm{~nm})$ (Figure 3$).^{26}$ The resultant materials not only contained internal pores that could be flooded with electrolyte, but also possessed core-shell Si-C nanowires, which are favorable for the fast diffusion of lithium ions and the transfer of electrons. ${ }^{26}$ As a consequence, this $\mathrm{Si} @ \mathrm{C}$ composite exhibited a capacity of $3163 \mathrm{mAh} \mathrm{g}^{-1}$ and stable cycle performance at $0.2 \mathrm{C}$ for lithium storage. This capacity was about 8 times higher than that of commercial graphite electrode $\left(\sim 300 \mathrm{mAh} \mathrm{g}^{-1}\right)$. More importantly, a capacity of $2462 \mathrm{mAh} \mathrm{g}^{-1}$ at the higher rate of $2 \mathrm{C}$ could be achieved, representing an excellent high-rate performance for lithium storage. ${ }^{26}$ Following this strategy, a series of $\mathrm{CCNMs}$ based on porous $\mathrm{Si}^{2} \mathrm{C}^{27}, \mathrm{Ge}-\mathrm{C}^{28}$ nanocomposites and core-shell $\mathrm{Sn}_{78} \mathrm{Ge}_{22}-\mathrm{C}^{29}, \mathrm{Si}_{90} \mathrm{Ge}_{10}-\mathrm{C}^{30}$ nanowires with high capacities above $1000 \mathrm{mAh} \mathrm{g}^{-1}$ were fabricated. 

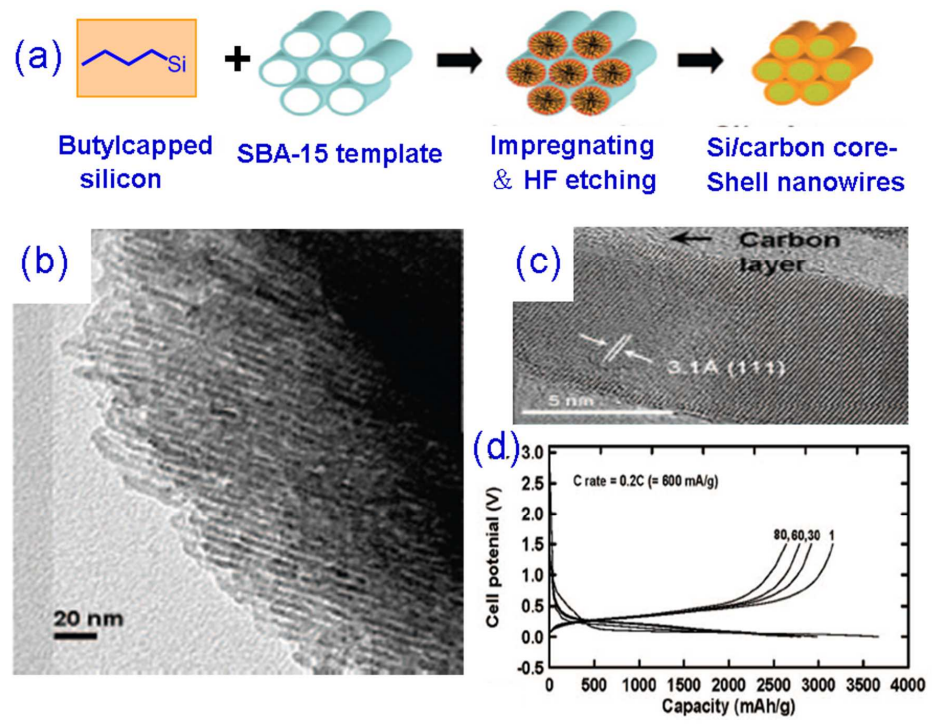

FIGURE 3. (a) Synthetic route to Si@carbon core-shell nanowires. (b) TEM and (c) HRTEM images of the Si@carbon nanowires. (d) Voltage profiles of the Si@carbon nanowire electrode at a rate of $0.2 \mathrm{C}^{26}$

Heteroatom (N, B and P)-enriched CCNMs have also been fabricated by pyrolysis of molecular precursors. ${ }^{31}$ The incorporation of such heteroatoms can be used to tailor the electronic properties and chemical reactivity of resulting materials, and thereby give rise to new functions, such as electrocatalysis of the oxygen reduction reaction (ORR) required in fuel cells. For instance, pyrolysis of nucleobases dissolved in an ionic liquid (1-ethyl-3-methylimidazolium dicyanamide), yielded a nitrogen-doped porous carbon material with surface area of up to 1500 $\mathrm{m}^{2} \mathrm{~g}^{-1}$ (Figure 4). ${ }^{32}$ Applying silica nanoparticles as a template in this process produced a nitrogen-doped CCNM with nitrogen content as high as $12 \mathrm{wt} \%$ and a narrow mesopore size distribution centered at $\sim 12 \mathrm{~nm}$. The as-prepared material was capable of catalyzing the four-electron transfer process required of the ORR. Moreover, the observed low onset voltage for 
oxygen reduction in alkaline medium and the high methanol tolerance of this CCNM are superior to those of commercial $\mathrm{Pt} / \mathrm{C}$ catalysts $^{32}$.

(a)

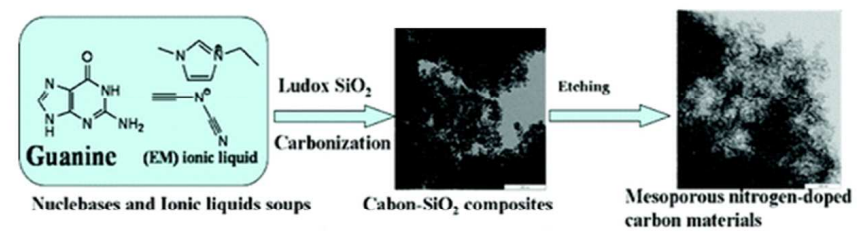

(b)

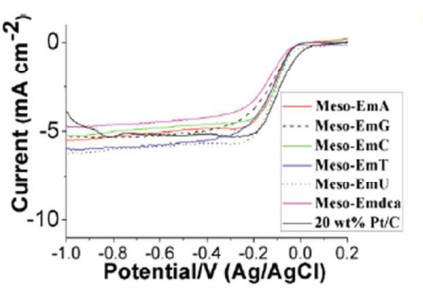

(c)

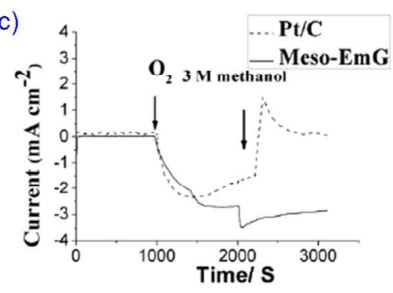

FIGURE 4. (a) Preparation of N-doped CCNMs as ORR catalysts. (b) RDE voltammograms of these materials. (c) Electrocatalytic selectivity among nitrogen, oxygen and methanol for the nitrogen-doped CCNM and Pt/carbon catalysts. ${ }^{32}$

\section{Aromatic polymer networks}

Polymer networks with a rigid molecular backbone and a relatively fixed 3D architecture, such as hyperbranched polyphenylenes or cross-linked conjugated polyphenylene-ethnylene networks, ${ }^{33-35}$ provide another route for the synthesis of porous CCNMs. One advantage of such precursors in relation to the molecular precursors discussed above is their higher carbonization yields. Moreover, polymer precursors employed in preparing CCNMs can themselves be conveniently tailored by variation of the monomers employed and/or via adjustment of polymerization protocols. This flexibility allows one to control both the chemical composition and the solid-state structure of the polymer precursors, and hence the final CCNMs, at the molecular level. ${ }^{15,20}$ 
(a)
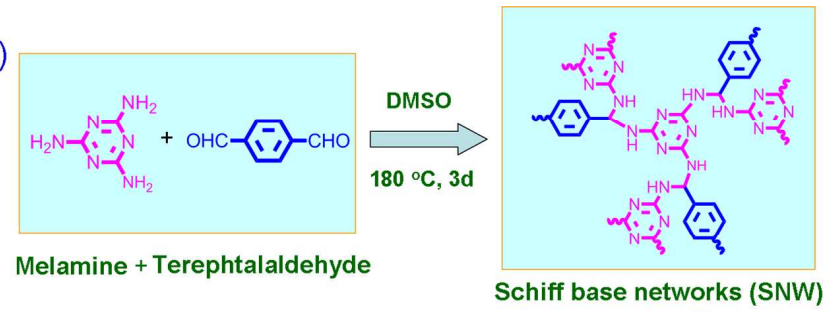

(b)
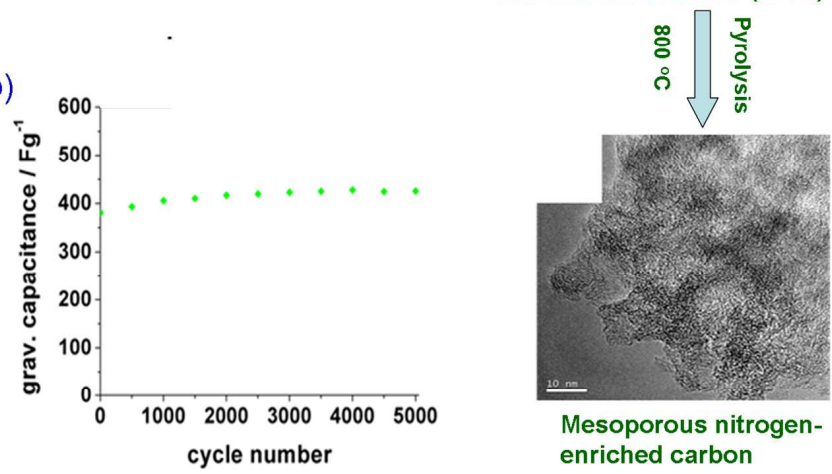

FIGURE 5. (a) Preparation of mesoporous nitrogen-enriched CCNMs. (b) Cycle performance of the $\mathrm{CCNM}$ in a $1 \mathrm{M} \mathrm{H}_{2} \mathrm{SO}_{4}$ electrolyte at a current density of $2 \mathrm{~A} \mathrm{~g}^{-1}$. ${ }^{16}$

For example, the one-pot A2B3-type polycondensation of melamine and terephtalaldehyde at $180^{\circ} \mathrm{C}$ in DMSO, generated a porous Schiff-base network. Subsequent pyrolysis of this network at $800^{\circ} \mathrm{C}$ provided a nitrogen-enriched CCNMs with mesoporosity, high surface area and tunable nitrogen content. ${ }^{16}$ This material exhibited a capacitance value of $381 \mathrm{~F} \mathrm{~g}^{-1}$ in an acid electrolyte and $351 \mathrm{~F} \mathrm{~g}^{-1}$ in an alkaline environment, with exceptionally stability over 5000 cycles (Figure $5)^{16}$. Both values are notably higher than those reported $\left(100-200 \mathrm{~F} \mathrm{~g}^{-1}\right)$ for similar materials produced by methods which do not generate mesoporosity. ${ }^{16}$ 
(a)
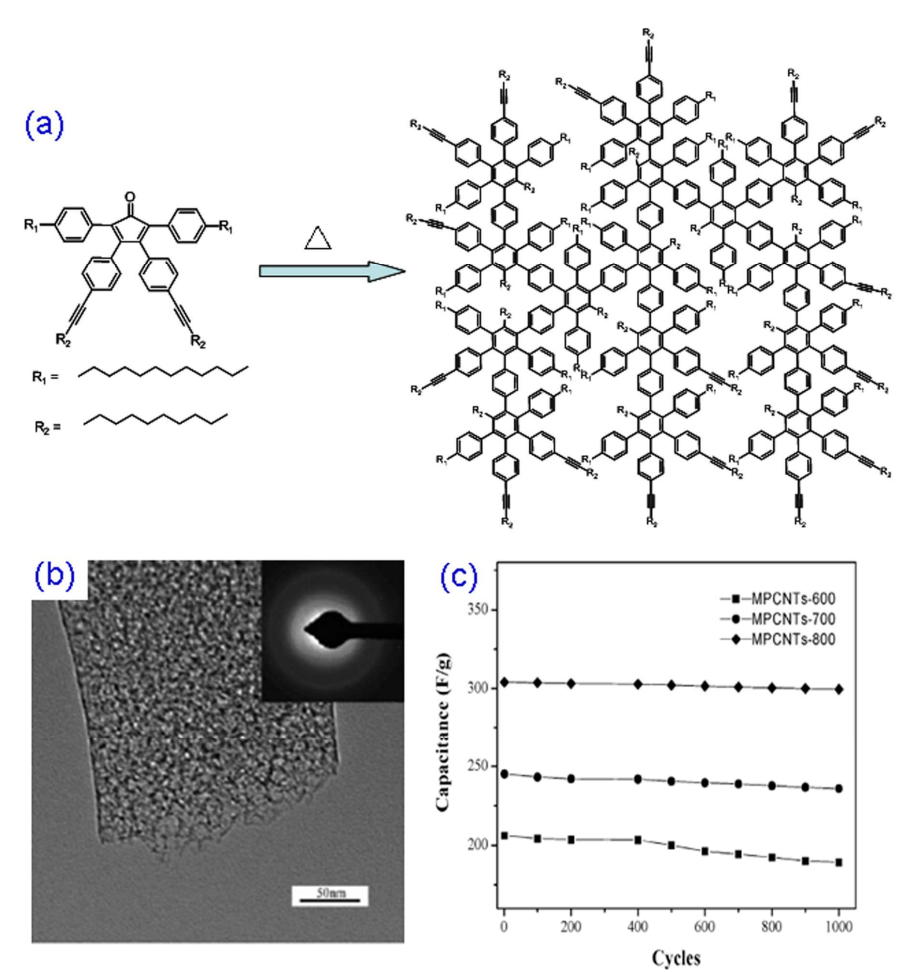

FIGURE 6. (a) Diels-Alder cycloaddition yielding a hyperbranched polyphenylene. (b) TEM image of the CCNM after pyrolysis. (c) Cycle performance of CCNMs from differing pyrolysis temperatures in $1 \mathrm{M} \mathrm{H}_{2} \mathrm{SO}_{4}$ electrolyte. $^{33}$

Another illustrative example is the pyrolysis of hyperbranched polyphenylene precursors obtained from an in situ thermally induced Diels-Alder reaction (Figure 6a). A typical AB2-type (one diene and two dienophiles) functionalized cyclopentadienone (CP) was impregnated into the nanochannels of an anodic aluminum oxide (AAO) membrane. Upon thermal annealing at $250^{\circ} \mathrm{C}$, alkyl-substituted hyperbranched polyphenylene was produced via intermolecular Diels-Alder reactions between the cyclopentadienone and alkyne moieties. Subsequent pyrolysis of the resulting polyphenylene precursor at $800^{\circ} \mathrm{C}$ generated a $\mathrm{CCNM}$ with $1 \mathrm{D}$ mesopores of between 10 and $20 \mathrm{~nm}$ and a surface area of $1140 \mathrm{~m}^{2} \mathrm{~g}^{-1} \cdot 33$ The resulting material exhibited a capacitance of $304 \mathrm{~F} \mathrm{~g}^{-1}$, with stable cycle performance in an acidic electrolyte (Figure 6). ${ }^{33}$ 
(a)

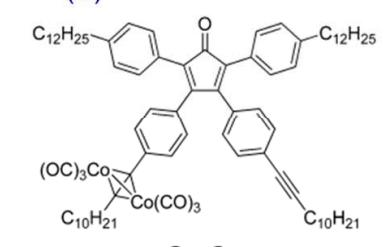

Co-Cp
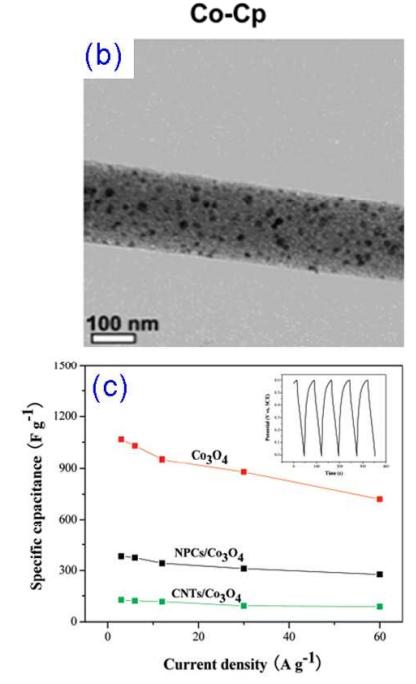

(d)
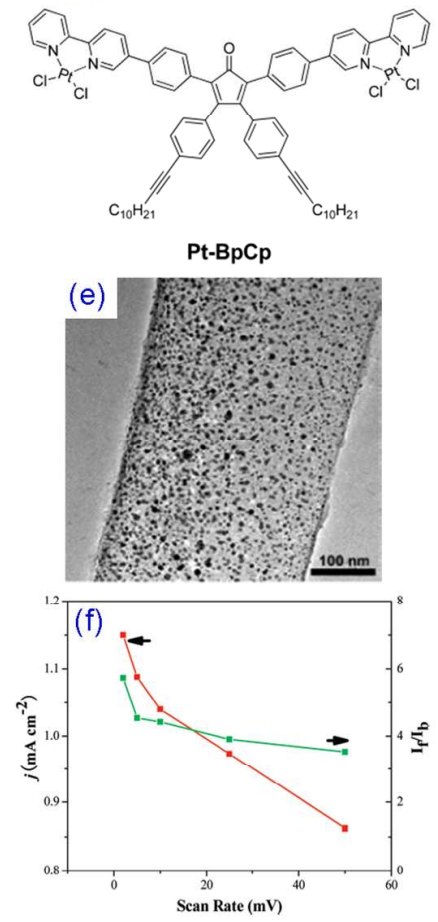

FIGURE 7. (a) Structure of Co-Cp. (b) TEM image of $\mathrm{C}^{-} \mathrm{Co}_{3} \mathrm{O}_{4}$. (c) Capacitance vs. current density curves of $\mathrm{C}-\mathrm{Co}_{3} \mathrm{O}_{4}, \mathrm{Co}_{3} \mathrm{O}_{4}$, and $\mathrm{CNTs} / \mathrm{Co}_{3} \mathrm{O}_{4}$ (inset: charge and discharge curves of C-Co $\mathrm{O}_{3} \mathrm{O}_{4}$ at a density of $6 \mathrm{~A} \mathrm{~g}^{-1}$ ). (d) Structure of Pt-BpCp. (e) TEM image of C-Pt. (f) Scan rate dependent current density $(j)$ and $I_{\mathrm{f}} / I_{\mathrm{b}}$ curves for the electro-oxidation of methanol on C-Pt. ${ }^{34}$

This strategy was extended to fabricate CCNMs by pyrolysis of polyphenylene precursors with integrated metal complex moieties. For instance, AB2-type 3,4-bis(4-dodecynylphenyl)-2,5-bis(4-dodecylphenyl)cyclopentadienone and its corresponding $\mathrm{Co}_{2}(\mathrm{CO})_{6}$ complex $(\mathrm{Co}-\mathrm{Cp}$, Figure $7 \mathrm{a})$ were used as monomers for the synthesis of cobalt functionalized polyphenylene skeletons via a Diels-Alder cycloaddition reactions. Pyrolysis of the resulting polymeric material produced a $1 \mathrm{D}$ porous CCNM consisting of amorphous carbon 
and $\mathrm{Co}_{3} \mathrm{O}_{4}$ nanocrystals $\left(\mathrm{C}-\mathrm{Co}_{3} \mathrm{O}_{4}\right){ }^{34}$ Similarly, a mixture of 3,4-bis(4-dodecynylphenyl)-2,5-bis(4-(2,2'-bipyridyl)phenyl) cyclopentadienone (BpCp) and its corresponding platinum dichloride complex (Pt-BpCp, Figure 7d) allowed the synthesis of a CCNM with Pt nanocrystals $(\mathrm{C}-\mathrm{Pt}) .{ }^{34}$ Structural characterizations revealed that the $\mathrm{Co}_{3} \mathrm{O}_{4}$ or $\mathrm{Pt}$ nanocrystals were homogeneously dispersed within the carbon matrix. Moreover, these carbon-metal (oxide) hybrids delivered excellent electrochemical performance for energy storage or conversion. As shown in Figure 7, use of $\mathrm{C}-\mathrm{Co}_{3} \mathrm{O}_{4}$ as an electrode material for a supercapacitor, yielded a gravimetric capacitance of $1066 \mathrm{~F} \mathrm{~g}^{-1}$, which ranks among the best electrochemical capacitive values for cobalt oxide electrode materials. ${ }^{34}$ Similarly, the C-Pt showed a higher catalytic efficiency toward methanol oxidation than commercial Pt-based materials when used as an electrocatalyst in fuel cells. ${ }^{34}$

\section{Self-assembling discotic molecules}

Discotic molecules, such as porphyrin ${ }^{36}$, perylene $^{37}$, hexa-peri-hexabenzocoronene $(\mathrm{HBC})^{21}$ and their derivatives, ${ }^{21}$ are a unique class of carbon-rich molecules capable of self-assembling into highly ordered columnar superstructures, and which frequently display liquid-crystalline phase behavior. As such, such systems can be thought of as intermediate between the low molecular weight molecular precursors and the polymer precursors discussed in the last two sections. Discotic molecules tend to orient themselves in an edge-on manner on substrates such as glass, polytetrafluoroethylene or highly oriented pyrolyzed graphite owing to their unique 
supramolecular behavior, that is, a configuration that preserves internal aromatic $\pi-\pi$ interactions at the expense of inhibited $\pi$-surface bonds. ${ }^{21,38}$ Consequently, pyrolysis of these discotic molecules typically results in the formation of graphitic carbon, as opposed to the amorphous materials discussed previously. ${ }^{7}$ For instance, pyrolysis of a branched alkyl substituted HBC at $600^{\circ} \mathrm{C}$, with $3 \mathrm{D}$ porous inverse silica opal employed as a template, produced monodisperse graphitic microbeads with an orderly alignment. ${ }^{17}$ More interestingly, thermal treatment of (HBC-PhC12) in an alumina membrane (Figure 8a-8c) afforded carbon nanotubes in which the graphitic layers are oriented perpendicular to the surface of tube axis. ${ }^{21}$ This concept was adapted to fabricate hollow carbon spheres by employing $\mathrm{HBC}-\mathrm{C} 12$ as the precursor and a silica/space/mesoporous-silica spheres as the template (Figure $8 \mathrm{~d}-8 \mathrm{f}$ ). ${ }^{7}$ The resulting hollow spheres exhibited uniform size and possessed dual walls, with a thicker, mesoporous exterior wall and thinner, solid interior wall. The nanochannels present in the exterior walls of the spheres are arranged perpendicularly to the surface spheres, which favors lithium ion diffusion, while the solid interior graphitic walls facilitate the collection and transport of electrons during electrochemical cycling (Figure 9). As a consequence, a reversible capacity (ca. $600 \mathrm{mAh} \mathrm{g}^{-1}$ ) and high-rate capability (about $200 \mathrm{mAh} \mathrm{g}^{-1}$ at the rate of $10 \mathrm{C}$ ) were achieved for lithium storage. $^{7}$ 

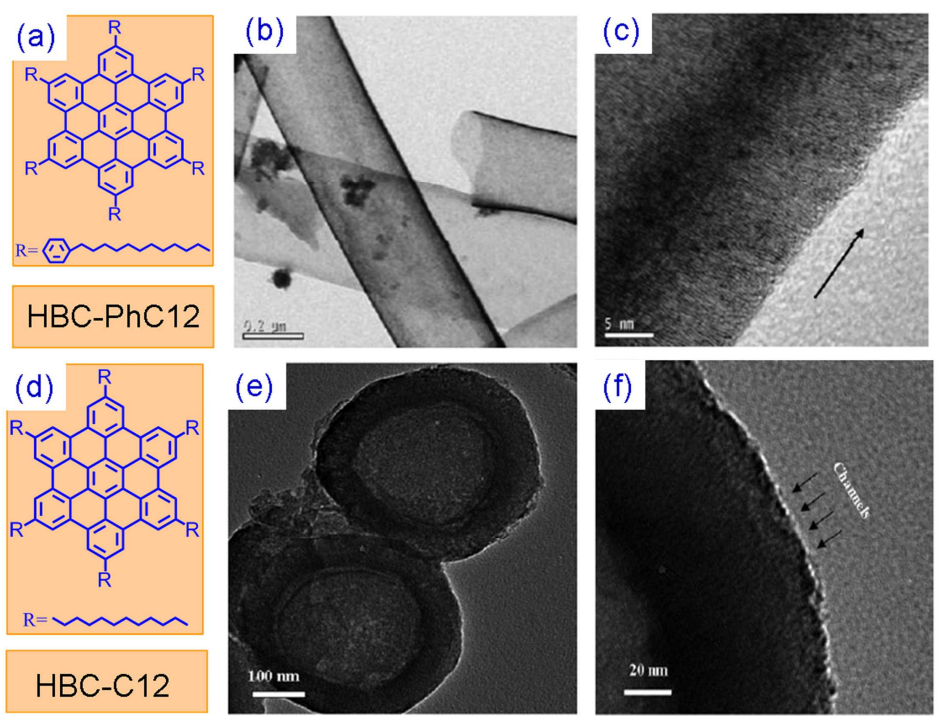

FIGURE 8. (a) Structure of HBC-Ph-C12. (b,c) HRTEM images of the CCNM showing the orientation of the graphitic layers in the tube wall. ${ }^{18}$ (d) Structure of HBC-C12. (e,f) HRTEM images of resulting hollow carbon spheres. The arrows denote the perpendicular arrangement of the surface nanochannels. ${ }^{7}$
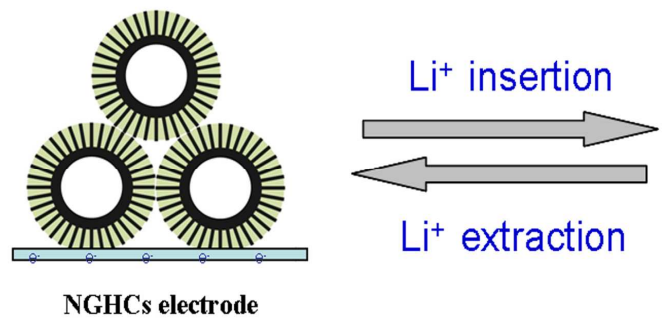

FIGURE 9. Diffusion of lithium ions and electrons during the electrochemical cycling of an electrode based on hollow carbon spheres produced from the templated pyrolysis of HBC-C12. ${ }^{7}$

Use of nitrogen-containing precursors, such as N,N'-bis(2,6-diisopropyphenyl)3,4,9,10-perylenetetracarboxylic diimide (PDI) or tetrakis(tert-butyl) naphthalocyanine, and templates such as ordered mesoporous silica SBA-15 allowed for the fabrication of nitrogen-enriched CCNMs incorporating a nitrogen content of $\sim 3 \mathrm{wt} \%$ in a mesoporous graphitic 
carbon matrix (Figure 10). ${ }^{39}$ These materials, which possess a surface area of $\sim 500 \mathrm{~m}^{2} \mathrm{~g}^{-1}$, function as a metal-free catalyst for the ORR, exhibiting high electrocatalytic activity and long-term stability in alkaline conditions. ${ }^{39}$ Indeed, the characteristics of these materials were superior to those observed for commercially available Pt-C catalysts in terms of current density and electron transfer number. Additionally, this work demonstrated the importance the presence of graphite-like nitrogen for the electrocatalytic activity. ${ }^{39}$

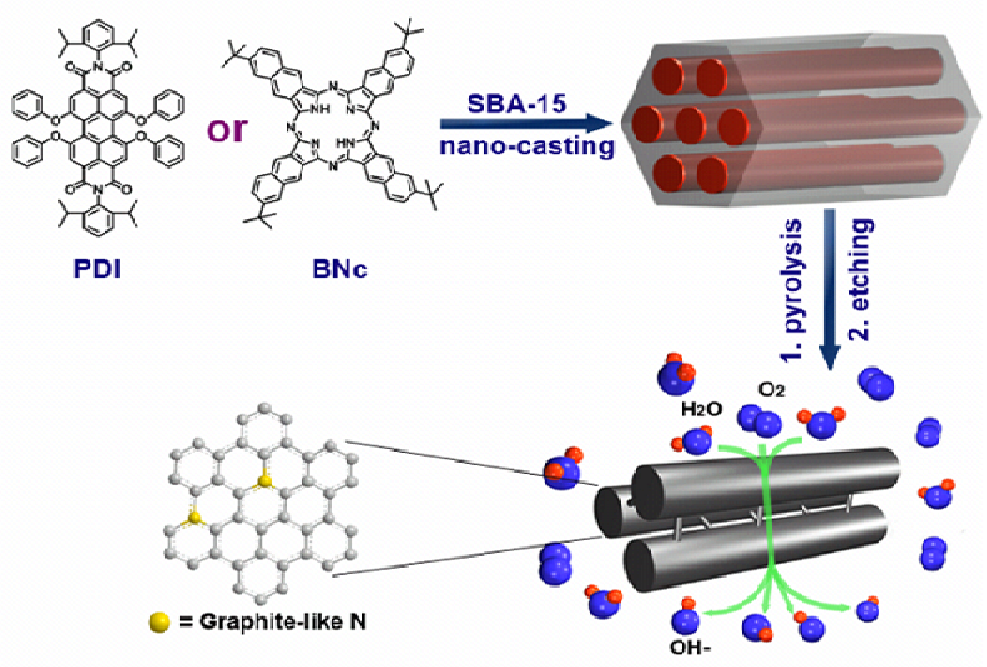

FIGURE 10. Preparation of nitrogen-enriched CCNMs as metal-free catalysts for ORR. ${ }^{39}$

\section{Graphene and graphene oxide sheets}

Graphene, a 2D "aromatic" monolayer of carbon atoms, has attracted tremendous attention recently owing to its host of attractive physical properties, including superior electrical conductivity, large surface area, excellent mechanical flexibility, and high thermal/chemical stability. $^{14,40-42}$ Various graphene-metal (oxide) composites such as graphene-Pt, ${ }^{43}$ graphene- $\mathrm{SnO}_{2}{ }^{44}$, graphene- $\mathrm{Co}_{3} \mathrm{O}_{4}{ }^{45}$ and graphene- $\mathrm{Mn}_{3} \mathrm{O}_{4}{ }^{46}$ have been reported. These materials 
show enhanced electrochemical properties with respect to the corresponding metal (oxide) systems by taking advantage of the additional properties provided by the graphene component. ${ }^{14,45,46}$ In particular, graphene can provide a fast electron-transport pathway in the overall electrode and accommodate the volume change of metal (oxide) during the electrochemical reactions. Nevertheless, graphene sheets commonly suffer from random stacking with metal (oxide) nanoparticles due to the intrinsic incompatibility of these two classes of materials and the tendency of both materials to self-aggregate. ${ }^{13}$ The undefined structure of graphene-based nanocomposites and the inhomogeneous dispersion of metals (oxides) in the graphene matrix usually result in a reduction of the resulting electrochemical performance including the reversible capacity and cycle stability. ${ }^{14,44}$ Therefore, fabrication of graphene-based hybrid materials with well-defined structures remains an important goal. Several strategies for the fabrication of graphene-based CCNMs with defined core-shell or sandwich-like architecture have been established recently. Moreover, the resulting materials show promise for application for energy storage and conversions. ${ }^{40}$

The first strategy employs graphene with tunable oxygen-containing groups to induce the growth of metal oxide or hydroxide nanocrystals. ${ }^{22,11,46,47}$ In 2010 , the first example of this approach demonstrated that the degree of oxidation of the graphene exerted a major influence on nanocrystal formation by metal hydroxides or oxides during hydrothermal processing. ${ }^{22,} 47$ Hence, tuning the surface chemistry of graphene substrates offers a way to control the morphology and crystallinity of the metal hydroxide/oxide nanocrystals incorporated into these systems, as well as the performance of the resulting CCNM (Figure 11). ${ }^{47}$ For example, growth 
of $\mathrm{Ni}(\mathrm{OH})_{2}$ on the lightly oxidized graphene sheets generated crystalline hexagonal nanoplates with uniform size. ${ }^{12}$ The resulting nanostructure allowed for fast charge transport between the active nanoplates and the graphene-based current collectors, resulting in a specific capacitance of $1335 \mathrm{~F} \mathrm{~g}^{-1}$ at a current density of $2.8 \mathrm{~A} \mathrm{~g}^{-1}$ and a stable cycling performance (Figure 11c). This performance, which is significantly better than the $339 \mathrm{~F} \mathrm{~g}^{-1}$ obtained for a mechanically mixed sample of graphene and $\mathrm{Ni}(\mathrm{OH})_{2}$, clearly demonstrates the impact that processing protocols can have on device performance. ${ }^{12}$

(a)

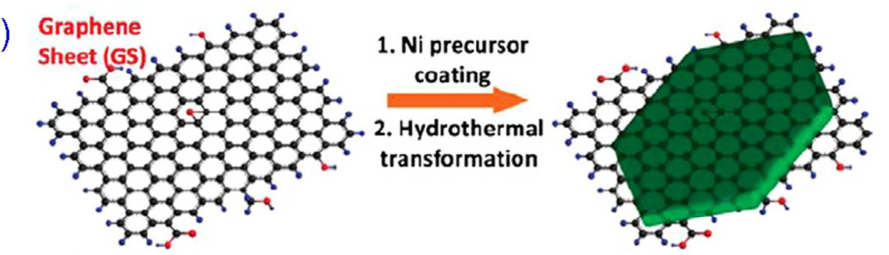

(b)

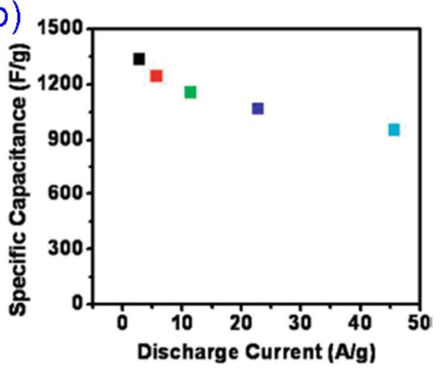

(c)

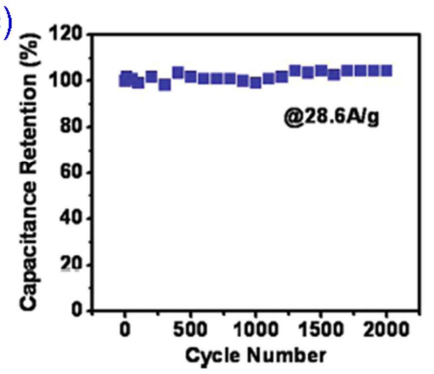

FIGURE 11. (a) Illustration of $\mathrm{Ni}(\mathrm{OH})_{2}$ nanocrystal growth on lightly oxidized graphene. ${ }^{42}$ (b) Capacitance of $\mathrm{Ni}(\mathrm{OH})_{2} /$ graphene at various discharge current densities. ${ }^{9}$ (c) Cycle performance of $\mathrm{Ni}(\mathrm{OH})_{2} /$ graphene at a current density of $28.6 \mathrm{~A} \mathrm{~g}^{-1} .12$

Recently, we also found that porous iron oxide ribbons could be fabricated by controlled growth of iron glycolate on the surface of poly(N-vinyl-2-pyrrolidone) (PVP)-functionalized graphene followed by a thermal annealing at $250^{\circ} \mathrm{C}$ in air (Figure $12 \mathrm{a}$ ). ${ }^{48}$ The resulting iron oxide 
ribbons possess a large aspect ratio and porous structure, providing numerous open channels allowing for access of electrolyte and thereby facilitating rapid diffusion of lithium ions from electrolyte to electrode. The observed morphology also accommodates the volume change of the iron oxides associated with lithium incorporation. As a result of these structural features, these hybrids exhibited a reversible capacity of $1050 \mathrm{mAh} \mathrm{g}^{-1}$ in the first 10 cycles, and over 1000 $\mathrm{mAh} \mathrm{g}^{-1}$ after 130 cycles (Figure $\left.12 \mathrm{~b}, 12 \mathrm{c}\right)^{48}$.

(a)
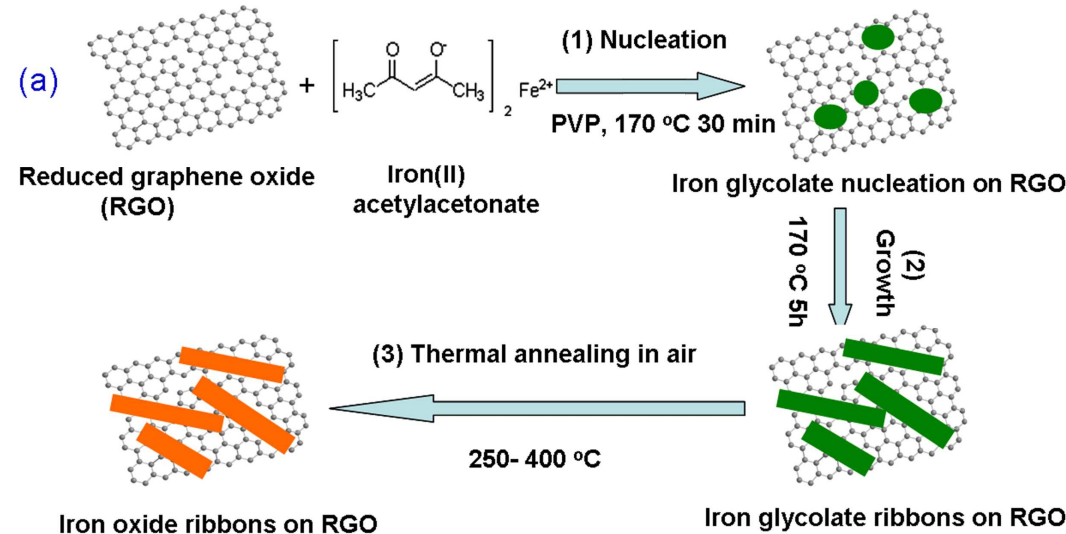

(b)
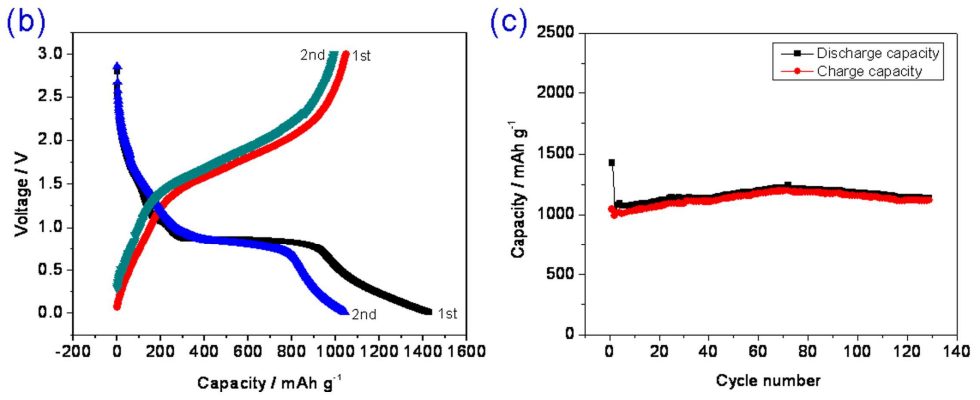

FIGURE 12. (a) Fabrication of of iron oxide ribbons. (b) Discharge-charge curves and (c) cycle performance of FeO-250 ribbons at a current density of $74 \mathrm{~mA} \mathrm{~g}^{-1}{ }^{48}$ 
(a)

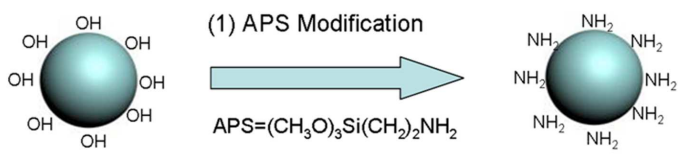

Metal oxide

APS modified metal oxide

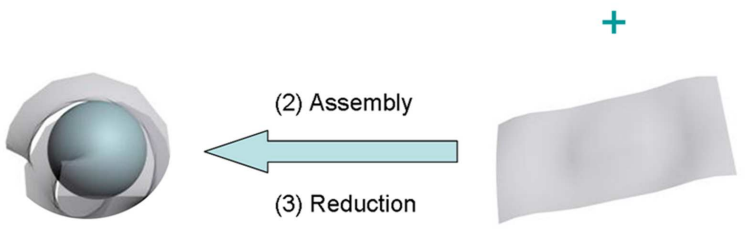

Graphene encapsulating metal oxide (GE-MO)

Graphene oxide

(GO)
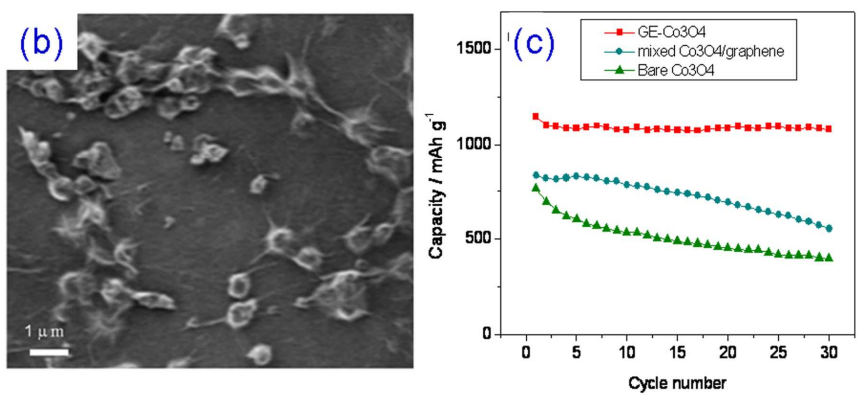

FIGURE 13. (a) Fabrication of graphene-encapsulated metal oxides. (b) TEM image of G- $\mathrm{Co}_{3} \mathrm{O}_{4}$ nanoparticles. (c) Comparison of the cycle performance of $\mathrm{G}-\mathrm{Co}_{3} \mathrm{O}_{4}$, mixed $\mathrm{Co}_{3} \mathrm{O}_{4} /$ graphene composite and bare $\mathrm{Co}_{3} \mathrm{O}_{4}$ electrodes at a current density of $74 \mathrm{~mA} \mathrm{~g}^{-1} \cdot{ }^{40}$

The second strategy relies on encapsulation of metal (oxide) nanoparticles within individual carbon-based shells in order to completely prevent aggregation of the metal (oxide) nanoparticles. A key challenge in such an approach is to achieve both a high electrical conductivity and a high-weight fraction of the active electrode materials. Given its combination of electrical conductivity and mechanical flexibility, graphene is an ideal material for the encapsulation of metal (oxide) nanoparticles to yield core-shell CCNMs. In principle, the graphene layer can simultaneously 1) suppress the aggregation of the nanoparticles; 2) 
accommodate the volume change of the metal (oxide) during the electrochemical cycling; 3 ) yield a high metal (oxide) content in the final composite; and 4) maintain a high electrical conductivity in the electrode.

A representative example of this approach is our recently reported fabrication of graphene-encapsulated $\mathrm{Co}_{3} \mathrm{O}_{4}$ nanoparticles $\left(\mathrm{G}^{-} \mathrm{Co}_{3} \mathrm{O}_{4}\right)$ via the co-assembly of negatively charged graphene oxide (GO) and positively charged $\mathrm{Co}_{3} \mathrm{O}_{4}$ nanoparticles, followed by chemical reduction of the GO (Figure 13a, 13b). ${ }^{40}$ This $\mathrm{G}-\mathrm{Co}_{3} \mathrm{O}_{4}$ composite showed a reversible capacity of $1100 \mathrm{mAh} \mathrm{g}^{-1}$ in the first 10 cycles, and stable cycle performance, retaining a capacity of $\sim 1000 \mathrm{mAh} \mathrm{g}^{-1}$ after 130 cycles (Figure 13c), which is 3 times higher than that of commercial graphite electrode $\left(\sim 300 \mathrm{mAh} \mathrm{g}^{-1}\right) .^{40}$ Following this strategy, various graphene-encapsulated metals and metal oxides ( $\mathrm{Si}, \mathrm{Sn}, \mathrm{Ge}, \mathrm{SnO}_{2}, \mathrm{Fe}_{2} \mathrm{O}_{3}$ ) are currently being investigated as materials for enhanced lithium storage applications.

The third strategy takes advantage of the 2D nature of graphene to allow for the synthesis of graphene-based CCNMs with a sandwich-like structure. The resulting composite nanosheets inherit the intrinsic features of graphene-high surface area, large aspect ratios, negligible thickness and enhanced electrical conductivity-which are attractive for energy-related applications. An example of this approach is the fabrication of graphene-based mesoporous silica (G-silica) nanosheets, in which graphene sheets are separated by mesoporous silica layers. The synthesis uses cetyltrimethyl ammonium bromide (CTAB)-modified graphene oxide sheets as a template and tetraethylorthosilicate as the silica precursor (Figure 14). The resulting G-silica nanosheets possess a large aspect ratio, mesoporous structure, high surface area, and high 
(a)

2) Hydrolysis of TEOS and removal of CTAB
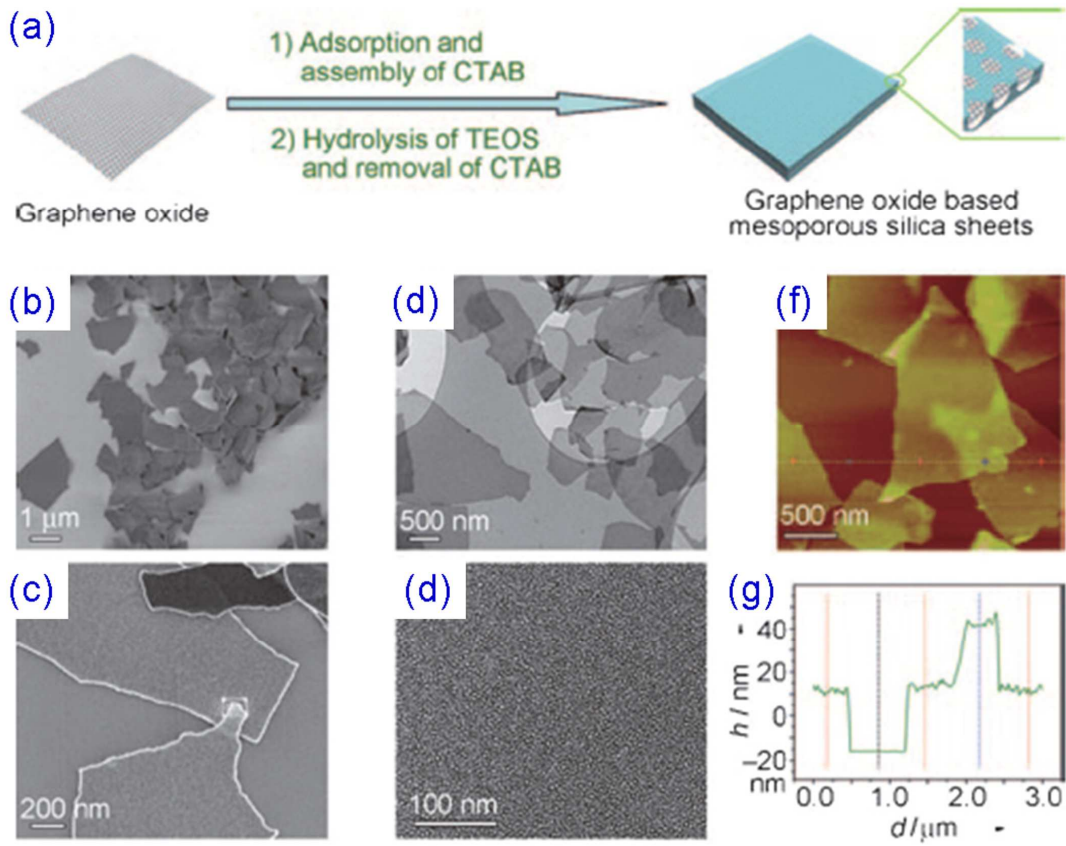

FIGURE 14. (a) Illustration of the fabrication of graphene oxide based mesoporous silica (G-silica) sheets, (b,c) FE-SEM and (d,e) TEM images of G-silica. (f) Representative AFM image and $(\mathrm{g})$ corresponding thickness analysis taken around the white line in (f). ${ }^{13}$

monodispersity. This approach also allows for the generation of composite nanosheets incorporating layers of materials like mesoporous carbon, metal, and metal oxide sheets on graphene. For instance, graphene based mesoporous titania $\left(\mathrm{G}-\mathrm{TiO}_{2}\right)$ nanosheets were fabricated by treating preformed G-silica nanosheets with $\left(\mathrm{NH}_{4}\right)_{2} \mathrm{TiF}_{6}{ }^{49}$ The numerous open channels in the resulting $\mathrm{G}_{-} \mathrm{TiO}_{2}$ allow access of electrolyte, facilitating the ultrafast diffusion of lithium ions during the cycling processes. Moreover, the graphene layer within each nanosheet acts as mini-current collectors, aiding fast electron transport in the electrode. As a result, a high-rate capability of $123 \mathrm{mAh} \mathrm{g}^{-1}$ at $10 \mathrm{C}$ and good cycle performance could be achieved for the $\mathrm{G}-\mathrm{TiO}_{2}$ 
nanosheets (Figure 15). For comparison, $\mathrm{TiO}_{2}$ nanosheets without graphene show a capacity of only approximately $40 \mathrm{mAh} \mathrm{g}^{-1} \cdot 49$
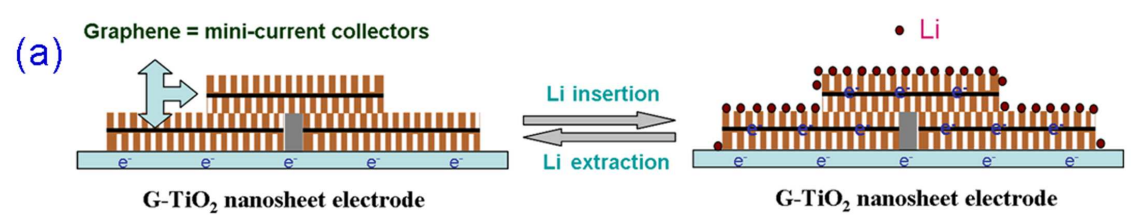

(b)

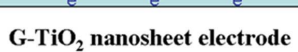
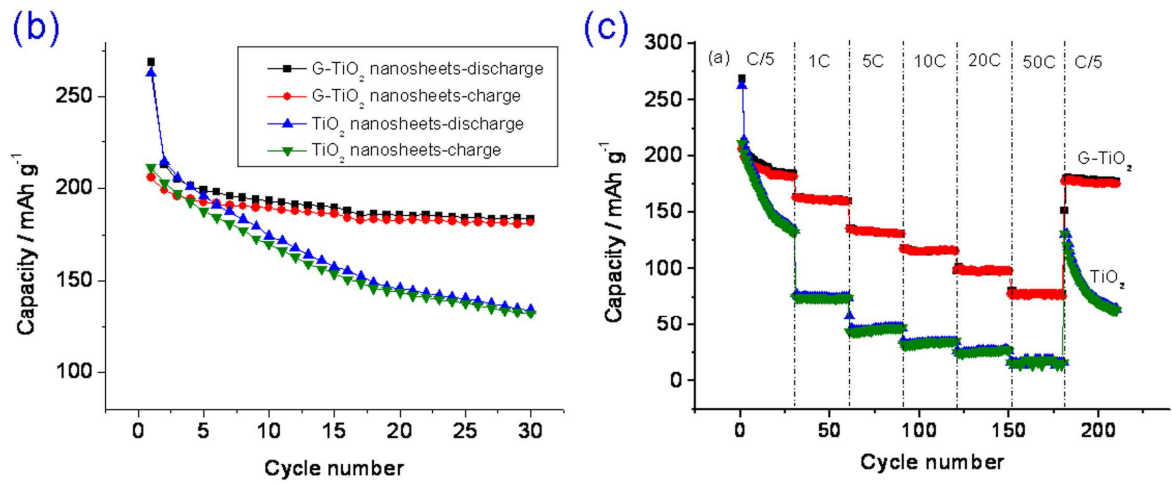

FIGURE 15. (a) Lithium insertion and extraction in $\mathrm{G}_{-} \mathrm{TiO}_{2}$ nanosheets,. (b) Initial charge-discharge curves of $\mathrm{G}_{-} \mathrm{TiO}_{2}$ nanosheets at a current density of $\mathrm{C} / 5$. (c) Cycle performance of $\mathrm{G}-\mathrm{TiO}_{2}$ and $\mathrm{TiO}_{2}$ nanosheets. ${ }^{49}$

\footnotetext{
Sandwich-like graphene carbon nitride $(\mathrm{G}-\mathrm{CN})$ nanosheets were produced from $\mathrm{G}-$-silica templates using ethylenediamine and carbon tetrachloride as the $\mathrm{CN}$ precursors (Figure 16). ${ }^{50}$ The resulting $\mathrm{G}-\mathrm{CN}$ nanosheets possessed a nitrogen content of approximately $20 \mathrm{wt} \%$, a thicknesses of about $18 \mathrm{~nm}$ and high surface area up to $542 \mathrm{~m}^{2} \mathrm{~g}^{-1} \cdot{ }^{50}$ These features allowed ready access of oxygen to the catalyst surface and facilitated the rapid diffusion of electrons through the electrode during the ORR, providing excellent electrocatalytic activity in alkaline
} 
solution. ${ }^{50}$ The current density, electron-transfer number, durability, and selectivity were superior to those observed for $\mathrm{CN}$ sheets without graphene or commercially available $\mathrm{Pt}-\mathrm{C}$ catalysts.

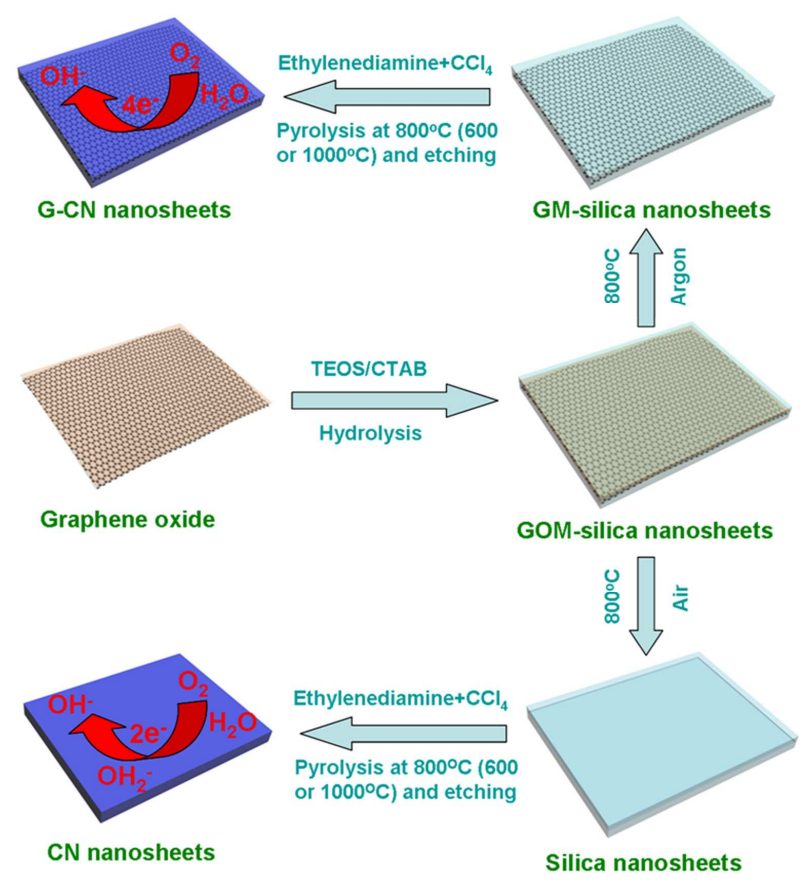

FIGURE 16. Fabrication of carbon nitride $(\mathrm{G}-\mathrm{CN})$ and $\mathrm{CN}$ nanosheets for the ORR. ${ }^{50}$

\section{Conclusions and Prospects}

In summary, CCNMs are playing an increasingly important role in improving the performance of electrochemical energy storage and conversion devices owing to their favorable nanoscale structural features and the intrinsic synergy between the two components of the systems. In this Account, we have summarized recent representative advancements toward the controlled synthesis of CCNMs as a function of the carbon precursor. The relationship between the structures of the resulting CCNMs and their electrochemical performance has also been highlighted in this context. Although a variety of CCNMs with tunable structures and 
compositions have been fabricated via the methods discussed, these results are only the beginning. It seems clear that the continued development of these approaches will yield new multifunctional CCNMs with enhanced electrochemical properties suitable for application in energy storage and conversion devices. It also seems reasonable that these materials could make miniaturized devices, such as nanoscale batteries, possible.

With regard to the three "molecular" approaches (low molecular weight organic or organometallic systems, aromatic polymer networks and discotic molecules), a deeper understanding regarding the relationship between the structure and physical properties of the precursors and those of the product CCNM will be required. However, even at this early stage a few initial conclusions seem warranted. For example, use of low molecular weight molecular precursors or aromatic polymer networks typically gives rise to an amorphous carbon component in the CCNM. In contrast, use of discotic precursors typically yields a graphitic matrix.

For the graphene-based hybrids, control of bulk morphology and the nanostructure of the other functional components on the graphene surface are critical issues. While the successful fabrication of graphene-based CCNMs including graphene-nanocrystal composites, core-shell graphene-encapsulated metal (oxide) nanoparticles and sandwich-like graphene-metal oxide nanosheets has been achieved, this area of research is still in its infancy and many challenges remain. In particular, the obstacle of the intrinsic incompatibility between graphene and inorganic materials needs to be resolved in order to gain fuller access to graphene-based CCNMs with desirable nanoscale architectures in a controlled manner. Given the rapid development of this research direction, one can expect that additional graphene-based hybrid materials 
incorporating a variety of multifunctional components will soon be readily available. Such an achievement will, in our view, result in a range of novel electrode materials for the next generation energy storage and conversion devices.

\section{ACKNOWLEDGMENT}

This work was financially supported by ERC grant on NANOGRAPH, the Max Planck Society through the program ENERCHEM, DFG Priority Program SPP 1459, BMBF LiBZ Project, and ESF Project GOSPEL (Ref Nr: 09-EuroGRAPHENE-FP-001), EU Project MOLESOL and GENIUS. REB acknowledges the fellowship support from the Alexander von Humboldt Foundation and the German Academic Exchange Service.

\section{BIOGRAPHICAL INFORMATION}

Dr. Shubin Yang received his PhD from Beijing University of Chemical Technology in 2008 after carrying out a thesis on high-performance lithium ion batteries under the guidance of Prof. Huaihe Song. And then he pursued postdoctoral research with Prof. K. Müllen at Max-Planck Institute for Polymer Research. His current research interests involve in carbon-rich materials for energy storage and conversions.

Prof. Robert E. Bachman received his $\mathrm{PhD}$ degree from Rice University in 1994 under the direction of Prof. Kenton Whitmire. After an Alexander von Humboldt (AvH) post-doctoral 
fellowship at the Technical University München with Prof. Hubert Schmidbaur, Prof. Bachman joined the faculty of Georgetown University in 1995. He moved to The University of the South in 2001 and was promoted to Professor in 2010. Prof. Bachman spent 2011-12 on sabbatical at the Max Planck Institute for Polymer Research. His current research interests focus on self-assembly of inorganic complexes and soft inorganic materials.

Prof. Xinliang Feng joined the group of Prof. K. Müllen at the Max Planck Institute for Polymer Research (MPIP) and obtained his PhD degree in April 2008. Since December 2007, he was appointed as project leader in MPIP. He became a Professor at Shanghai Jiao Tong University in 2011. His current scientific interests include the conjugated oligomers and polymers, graphene, carbon-rich molecules and materials for electronic and energy-related applications.

Prof. Klaus Müllen received his $\mathrm{PhD}$ in 1972 at the University of Basel (Switzerland). He pursued postdoctoral research with Prof. J.F.M. Oth at ETH Zurich, where he obtained his habilitation in 1977. After working as a Professor of Organic Chemistry at the universities of Cologne and Mainz, he became a scientific member of the Max Planck Society in 1989 and was appointed Director of the Department for Synthetic Chemistry at MPIP. His current research focus lies on synthetic macromolecular chemistry and materials science.

\section{Corresponding Author}

*muellen@mpip-mainz.mpg.de, feng@mpip-mainz.mpg.de

\section{Notes}


The authors declare no competing financial interest

\section{RERENCES}

1. Maier, J., Nanoionics: ion transport and electrochemical storage in confined systems. Nature Mater. 2005, 4 (11), 805-815.

2. Guo, Y. G.; Hu, J. S.; Wan, L. J., Nanostructured materials for electrochemical energy conversion and storage devices. Adv. Mater. 2008, 20 (15), 2878-2887.

3. Recham, N.; Chotard, J. N.; Dupont, L.; Delacourt, C.; Walker, W.; Armand, M.; Tarascon, J. M., A 3.6 V lithium-based fluorosulphate insertion positive electrode for lithium-ion batteries. Nature Mater. 2010, $9(1), 68-74$.

4. Taberna, L.; Mitra, S.; Poizot, P.; Simon, P.; Tarascon, J. M., High rate capabilities Fe3O4-based Cu nano-architectured electrodes for lithium-ion battery applications. Nature Mater. 2006, 5 (7), 567-573.

5. Magasinski, A.; Dixon, P.; Hertzberg, B.; Kvit, A.; Ayala, J.; Yushin, G., High-performance lithium-ion anodes using a hierarchical bottom-up approach. Nature Mater. 2010, 9 (4), 353-358.

6. Wessells, C. D.; Huggins, R. A.; Cui, Y., Copper hexacyanoferrate battery electrodes with long cycle life and high power. Nature Commun. 2011, 2. 
7. Yang, S. B.; Feng, X. L.; Zhi, L. J.; Cao, Q. A.; Maier, J.; Müllen, K., Nanographene-Constructed Hollow Carbon Spheres and Their Favorable Electroactivity with Respect to Lithium Storage. Adv. Mater. 2010, 22 (7), 838-842.

8. Hou, J. B.; Shao, Y. Y.; Ellis, M. W.; Moore, R. B.; Yi, B. L., Graphene-based electrochemical energy conversion and storage: fuel cells, supercapacitors and lithium ion batteries. PCCP 2011, 13 (34), 15384-15402.

9. Zhang, H. L.; Morse, D. E., Kinetically controlled catalytic synthesis of highly dispersed metal-in-carbon composite and its electrochemical behavior. J. Mater. Chem. 2009, 19 (47), 9006-9011.

10. Yang, S. B.; Song, H. H.; Chen, X. H., Electrochemical performance of expanded mesocarbon microbeads as anode material for lithium-ion batteries. Electrochem. Commun. 2006, 8 (1), 137-142.

11. Qu, Q. T.; Yang, S. B.; Feng, X. L., 2D Sandwich-like Sheets of Iron Oxide Grown on Graphene as High Energy Anode Material for Supercapacitors. Adv. Mater. 2011, 23 (46), 5574-5580.

12. Wang, H. L.; Casalongue, H. S.; Liang, Y. Y.; Dai, H. J., Ni(OH)(2) Nanoplates Grown on Graphene as Advanced Electrochemical Pseudocapacitor Materials. J. Am. Chem. Soc. 2010, 132 (21), 7472-7477.

13. Yang, S. B.; Feng, X. L.; Wang, L.; Tang, K.; Maier, J.; Müllen, K., Graphene-Based Nanosheets with a Sandwich Structure. Angew. Chem. Int. Ed. 2010, 49 (28), 4795-4799. 
14. Yang, S. B.; Cui, G. L.; Pang, S. P.; Cao, Q.; Kolb, U.; Feng, X. L.; Maier, J.; Müllen, K., Fabrication of Cobalt and Cobalt Oxide/Graphene Composites: Towards High-Performance Anode Materials for Lithium Ion Batteries. Chemsuschem 2010, 3 (2), 236-239.

15. Zhi, L. J.; Müllen, K., A bottom-up approach from molecular nanographenes to unconventional carbon materials. J. Mater. Chem. 2008, 18 (13), 1472-1484.

16. Liang, Y. Y.; Schwab, M. G.; Shu, J.; Graf, R.; Spiess, H. W.; Feng, X. L.; Müllen, K., Template-Free Fabrication of Nitrogen-Enriched Mesoporous Carbons from Schiff Base Networks and Applications in High-Performance Electrochemical Capacitors. Nature Mater.2012 (SUBMITTED).

17. Zhi, L.; Wang, J. J.; Cui, G. L.; Kastler, M.; Schmaltz, B.; Kolb, U.; Jonas, U.; Müllen, K., From well-defined carbon-rich precursors to monodisperse carbon particles with hierarchic structures. Adv. Mater. 2007, 19 (14), 1849-1853.

18. Cui, G. L.; Gu, L.; Zhi, L. J.; Kaskhedikar, N.; van Aken, P. A.; Müllen, K.; Maier, J., A Germanium-Carbon Nanocomposite Material for Lithium Batteries. Adv. Mater. 2008, 20 (16), 3079-3083.

19. Cui, G. L.; Hu, Y. S.; Zhi, L. J.; Wu, D. Q.; Lieberwirth, I.; Maier, J.; Müllen, K., A one-step approach towards carbon-encapsulated hollow tin nanoparticles and their application in lithium batteries. Small 2007, 3 (12), 2066-2069. 
20. Schwab, M. G.; Fassbender, B.; Spiess, H. W.; Thomas, A.; Feng, X. L.; Müllen, K., Catalyst-free Preparation of Melamine-Based Microporous Polymer Networks through Schiff Base Chemistry. J. Am. Chem. Soc. 2009, 131 (21), 7216-7217.

21. Zhi, L. J.; Wu, J. S.; Li, J. X.; Kolb, U.; Müllen, K., Carbonization of disclike molecules in porous alumina membranes: Toward carbon nanotubes with controlled graphene-layer orientation. Angew. Chem. Int. Ed. 2005, 44 (14), 2120-2123.

22. Wang, H. L.; Yang, Y.; Liang, Y. Y.; Cui, L. F.; Casalongue, H. S.; Li, Y. G.; Hong, G. S.; Cui, Y.; Dai, H. J., LiMn(1-x)Fe(x)PO(4) Nanorods Grown on Graphene Sheets for Ultrahigh-Rate-Performance Lithium Ion Batteries. Angew. Chem. Int. Ed. 2011, 50 (32), 7364-7368.

23. Cui, G. L.; Zhi, L. J.; Thomas, A.; Lieberwirth, I.; Kolb, U.; Müllen, K., A novel approach towards carbon-Ru electrodes with mesoporosity for supercapacitors. Chemphyschem 2007, 8 (7), 1013-1015.

24. Kim, H.; Popov, B. N., Characterization of hydrous ruthenium oxide/carbon nanocomposite supercapacitors prepared by a colloidal method. J. Power Sources 2002, 104 (1), 52-61.

25. Zhi, L. J.; Hu, Y. S.; El Hamaoui, B.; Wang, X.; Lieberwirth, I.; Kolb, U.; Maier, J.; Müllen, K., Precursor-controlled formation of novel carbon/metal and carbon/metal oxide nanocomposites. Adv. Mater. 2008, 20 (9), 1727-1731.

26. Kim, H.; Cho, J., Superior Lithium Electroactive Mesoporous Si@Carbon Core-Shell Nanowires for Lithium Battery Anode Material. Nano Lett. 2008, 8 (11), 3688-3691. 
27. Kim, H.; Han, B.; Choo, J.; Cho, J., Three-Dimensional Porous Silicon Particles for Use in High-Performance Lithium Secondary Batteries. Angew. Chem. Int. Ed. 2008, 47 (52), 10151-10154.

28. Seo, M. H.; Park, M.; Lee, K. T.; Kim, K.; Kim, J.; Cho, J., High performance Ge nanowire anode sheathed with carbon for lithium rechargeable batteries. Energy Environ. Sci. 2011, 4 (2), 425-428.

29. Lee, H.; Cho, J., Sn(78)Ge(22)@carbon core-shell nanowires as fast and high-capacity lithium storage media. Nano Lett. 2007, 7 (9), 2638-2641.

30. Kwon, Y.; Kim, H.; Doo, S. G.; Cho, J. H., Sn0.9Si0.1/carbon core-shell nanoparticles for high-density lithium storage materials. Chem. Mater. 2007, 19 (5), 982-986.

31. Lee, J. S.; Wang, X. Q.; Luo, H. M.; Baker, G. A.; Dai, S., Facile Ionothermal Synthesis of Microporous and Mesoporous Carbons from Task Specific Ionic Liquids. J. Am. Chem. Soc. 2009, 131 (13), 4596-4597.

32. Yang, W.; Fellinger, T. P.; Antonietti, M., Efficient Metal-Free Oxygen Reduction in Alkaline Medium on High-Surface-Area Mesoporous Nitrogen-Doped Carbons Made from Ionic Liquids and Nucleobases. J. Am. Chem. Soc. 2011, 133 (2), 206-209.

33. Liang, Y. Y.; Feng, X. L.; Zhi, L. J.; Kolb, U.; Müllen, K., A simple approach towards one-dimensional mesoporous carbon with superior electrochemical capacitive activity. Chem. Commun. 2009, 7, 809-811. 
34. Liang, Y. Y.; Schwab, M. G.; Zhi, L. J.; Mugnaioli, E.; Kolb, U.; Feng, X. L.; Müllen, K., Direct Access to Metal or Metal Oxide Nanocrystals Integrated with One-Dimensional Nanoporous Carbons for Electrochemical Energy Storage. J. Am. Chem. Soc. 2010, 132 (42), 15030-15037.

35. Feng, X. L.; Liang, Y. Y.; Zhi, L. J.; Thomas, A.; Wu, D. Q.; Lieberwirth, I.; Kolb, U.; Müllen, K., Synthesis of Microporous Carbon Nanofibers and Nanotubes from Conjugated Polymer Network and Evaluation in Electrochemical Capacitor. Adv. Funct. Mater. 2009, 19 (13), 2125-2129.

36. Segade, A.; Castella, M.; Lopez-Calahorra, F.; Velasco, D., Synthesis and characterization of unsymmetrically beta-substituted porphyrin liquid crystals: Influence of the chemical structure on the mesophase ordering. Chem. Mater. 2005, 17 (21), 5366-5374.

37. Sienkowska, M. J.; Monobe, H.; Kaszynski, P.; Shimizu, Y., Photoconductivity of liquid crystalline derivatives of pyrene and carbazole. J. Mater. Chem. 2007, 17 (14), 1392-1398.

38. Wu, J. S.; Watson, M. D.; Zhang, L.; Wang, Z. H.; Müllen, K., Hexakis(4-iodophenyl)-peri-hexabenzocoronene - A versatile building block for highly ordered discotic liquid crystalline materials. J. Am. Chem. Soc. 2004, 126 (1), 177-186.

39. Liu, R. L.; Wu, D. Q.; Feng, X. L.; Müllen, K., Nitrogen-Doped Ordered Mesoporous Graphitic Arrays with High Electrocatalytic Activity for Oxygen Reduction. Angew. Chem. Int. Ed. 2010, 49 (14), $2565-2569$. 
40. Yang, S. B.; Feng, X. L.; Ivanovici, S.; Müllen, K., Fabrication of Graphene-Encapsulated Oxide Nanoparticles: Towards High-Performance Anode Materials for Lithium Storage. Angew. Chem. Int. Ed. 2010, 49 (45), 8408-8411.

41. Novoselov, K. S.; Geim, A. K.; Morozov, S. V.; Jiang, D.; Katsnelson, M. I.; Grigorieva, I. V.; Dubonos, S. V.; Firsov, A. A., Two-dimensional gas of massless Dirac fermions in graphene. Nature 2005, 438 (7065), 197-200.

42. El-Kady, M. F.; Strong, V.; Dubin, S.; Kaner, R. B., Laser Scribing of High-Performance and Flexible Graphene-Based Electrochemical Capacitors. Science 2012, 335 (6074), 1326-1330.

43. Yoo, E.; Okata, T.; Akita, T.; Kohyama, M.; Nakamura, J.; Honma, I., Enhanced Electrocatalytic Activity of Pt Subnanoclusters on Graphene Nanosheet Surface. Nano Lett. 2009, 9 (6), 2255-2259.

44. Paek, S. M.; Yoo, E.; Honma, I., Enhanced Cyclic Performance and Lithium Storage Capacity of $\mathrm{SnO}(2) /$ Graphene Nanoporous Electrodes with Three-Dimensionally Delaminated Flexible Structure. Nano Lett. 2009, 9 (1), 72-75.

45. Wu, Z. S.; Ren, W. C.; Wen, L.; Gao, L. B.; Zhao, J. P.; Chen, Z. P.; Zhou, G. M.; Li, F.; Cheng, H. M., Graphene Anchored with $\mathrm{Co}(3) \mathrm{O}(4)$ Nanoparticles as Anode of Lithium Ion Batteries with Enhanced Reversible Capacity and Cyclic Performance. Acs Nano 2010, 4 (6), 3187-3194.

46. Wang, H. L.; Cui, L. F.; Yang, Y. A.; Casalongue, H. S.; Robinson, J. T.; Liang, Y. Y.; Cui, Y.; Dai, H.

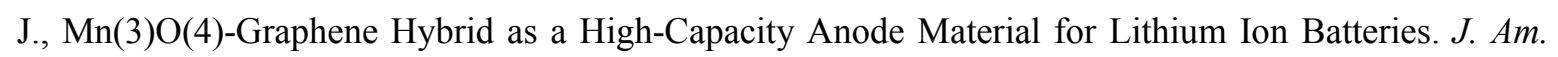
Chem. Soc. 2010, 132 (40), 13978-13980. 
1

2

3

4

5

6

7

8

9

10

11

12

13

14

15

16

17

18

19

20

21

22

23

24

25

26

27

28

29

30

31

32

33

34

35

36

37

38

39

40

41

42

43

44

45

46

47

48

49

50

51

52

53

54

55

56

57

58

59

60
47. Wang, H. L.; Robinson, J. T.; Diankov, G.; Dai, H. J., Nanocrystal Growth on Graphene with Various Degrees of Oxidation. J. Am. Chem. Soc. 2010, 132 (10), 3270-3271.

48. Yang, S. B.; Sun, Y.; Chen, L.; Hernandez, Y.; Feng, X. L.; Müllen, K., Porous Iron Oxide Ribbons Grown on Graphene for High-Performance Lithium Storage. Scientific Report 2012, 2, 427

49. Yang, S. B.; Feng, X. L.; Müllen, K., Sandwich-Like, Graphene-Based Titania Nanosheets with High Surface Area for Fast Lithium Storage. Adv. Mater. 2011, 23 (31), 3575-3579.

50. Yang, S. B.; Feng, X. L.; Wang, X. C.; Müllen, K., Graphene-Based Carbon Nitride Nanosheets as Efficient Metal-Free Electrocatalysts for Oxygen Reduction Reactions. Angew. Chem. Int. Ed. 2011, 50 (23), 5339-5343. 